\title{
Remotely Triggered Liquefaction of Hydrogel
}

\section{Materials}

Søren L. Pedersen, ${ }^{1}$ Tin H. Huynh, ${ }^{1}$ Philipp Pöschko, ${ }^{1}$ Anne Sofie Fruergaard, ${ }^{1}$

Morten T. Jarlstad Olesen, ${ }^{1}$ Yaqing Chen, ${ }^{1,2}$ Henrik Birkedal, ${ }^{1,2}$ Guruprakash Subbiahdoss, ${ }^{3}$ Erik Reimhult, ${ }^{3}$ Jan Thøgersen, ${ }^{1}$ Alexander N. Zelikin ${ }^{1,2}{ }^{*}$

1. Department of Chemistry, Aarhus University, Aarhus 8000, Denmark.

E-mail: zelikin@.chem.au.dk

2. iNano Interdisciplinary Nanoscience Centre, Aarhus University, Aarhus 8000, Denmark

3. Department of Nanobiotechnology, University of Natural Resources and Life Sciences, A1190 Vienna, Austria

\section{SUPPORTING INFORMATION}




\section{Supporting figures}
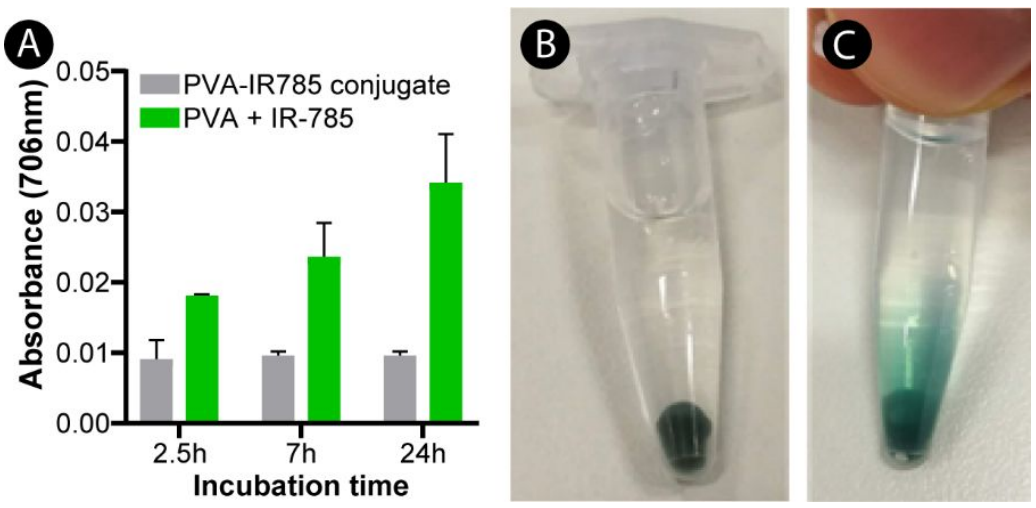

Figure S1: Spontaneous release of the non-conjugated NIR785. Hydrogels, $20 \mu \mathrm{L}$ volume, 5 cryocycles, $20 \mathrm{wt} \%$ of PVA-NIR785 or PVA-NH $\mathrm{NH}_{2}$ supplemented with equal amount of NIR785 in its free form. Hydrogels were incubated with $0.5 \mathrm{~mL}$ PBS at $37^{\circ} \mathrm{C}$. A) Absorbance of released NIR785 (free or as PVA-NIR785) to the supernatant over time. Results are plotted as mean + S.D., $n=3$ B) Picture of PVA-NIR785 hydrogel after 24h. C) Picture of PVA-NH $\mathrm{N}_{2} / \mathrm{NIR} 785$ hydrogel after $24 \mathrm{~h}$. 
A

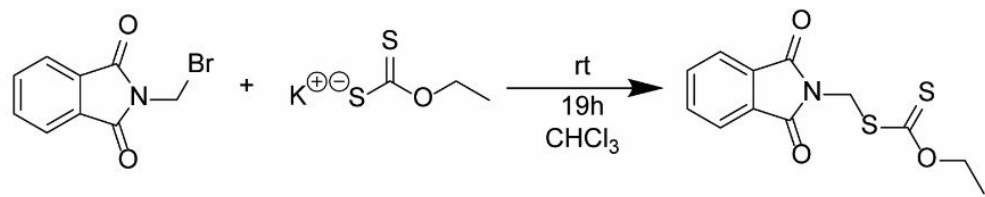

B

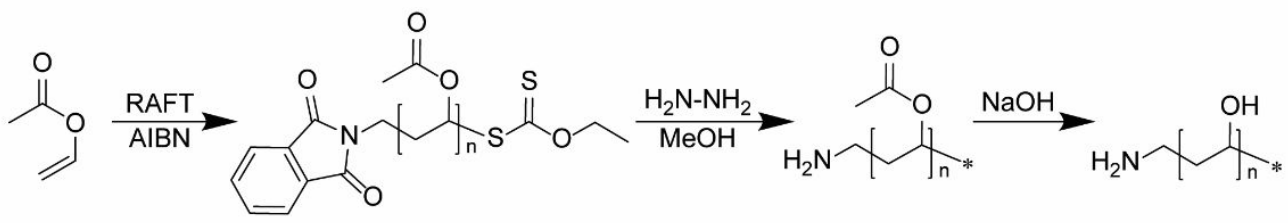

C<smiles>Cl/N=C/C=C/C=C/Nc1ccccc1</smiles>

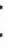
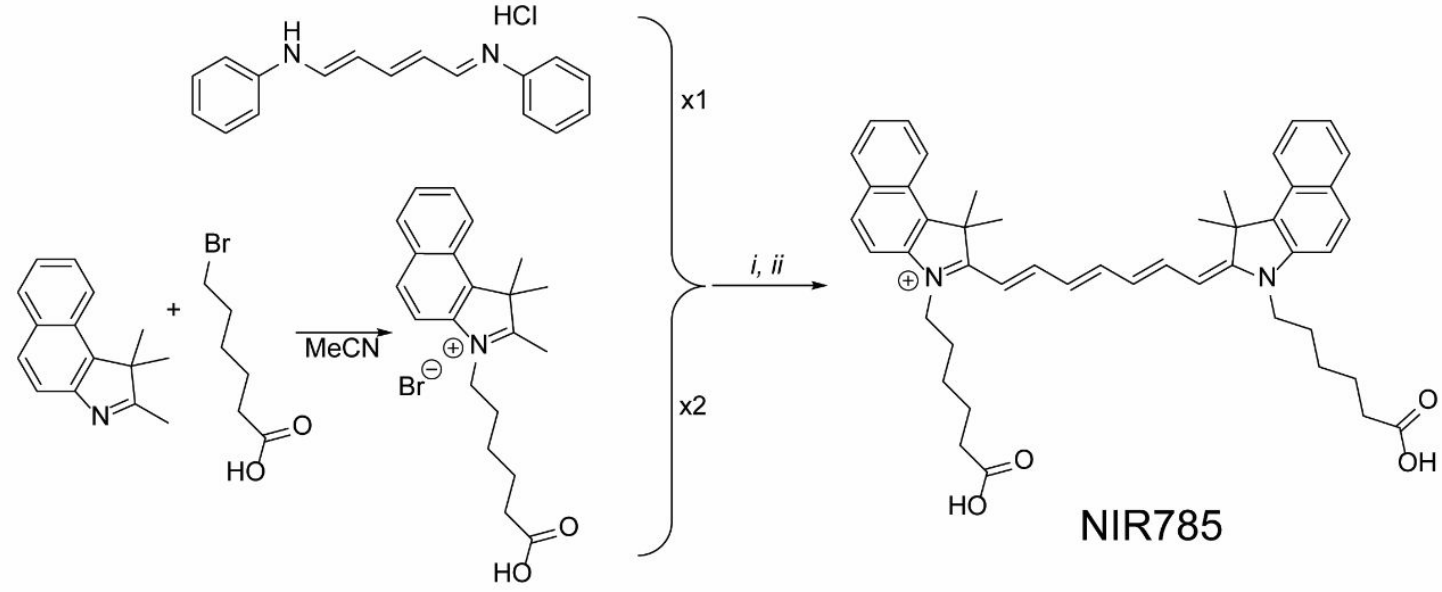

D
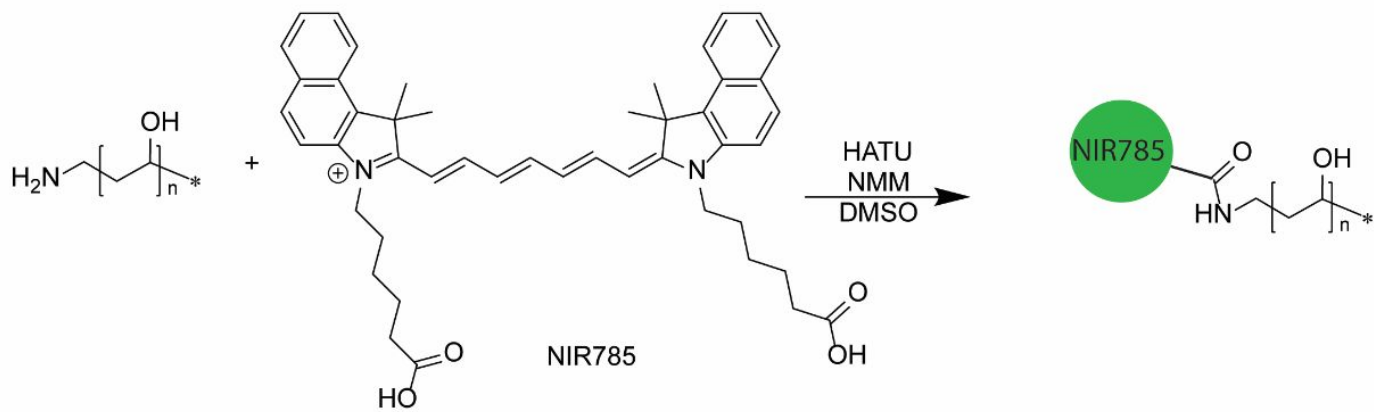

Figure S2: Schematic of the synthesis of PVA-NIR785. A) synthesis of phthalimide-containing chain transfer reagent. B) Synthesis of poly(vinyl acetate) followed by deprotection of acetate groups and the phthalimide functionality afforded PVA with one terminal amine group. C) Synthesis of NIR785. $i$ : $\mathrm{AcO}_{2}$, DIPEA, DCM. ii: AcONa, MeOH. D) Conjugation of PVA with NIR785 yielding PVANIR785. 


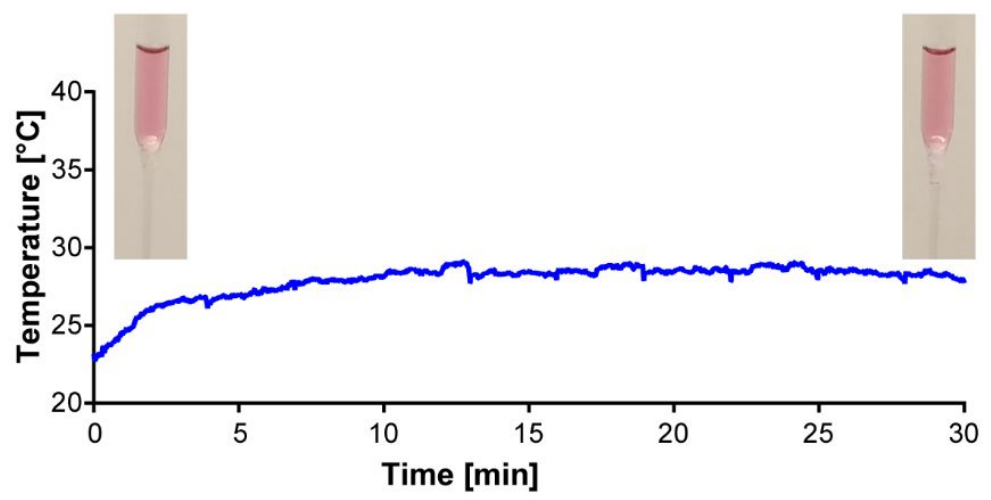

Figure S3: Stability of PVA hydrogels. PVA hydrogel $(50 \mu \mathrm{L}, 8 \mathrm{wt} \%$ PVA, $90 \mathrm{kDa})$ without AuNP irradiated by NIR light at $1.5 \mathrm{~W}$ for $30 \mathrm{~min}$. Temperature was recorded with an IR-camera. Inserts: picture at $\mathrm{t}=0$ and $\mathrm{t}=30 \mathrm{~min}$.

A

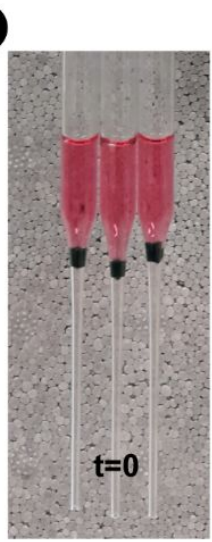

$37^{\circ} \mathrm{C}$

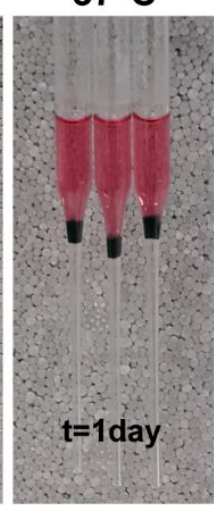

B

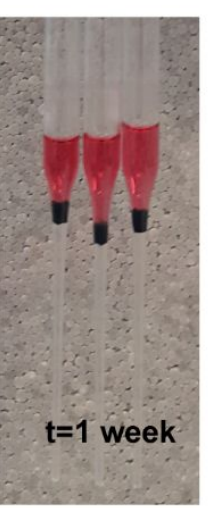

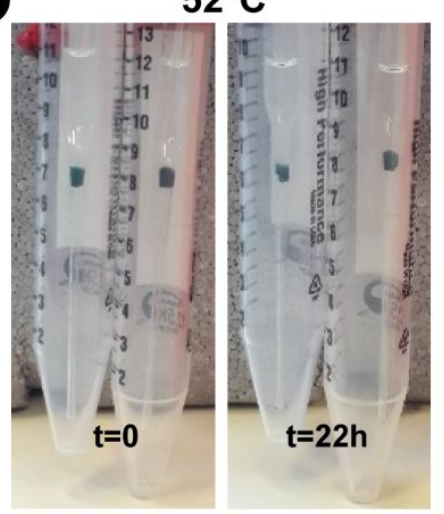

C

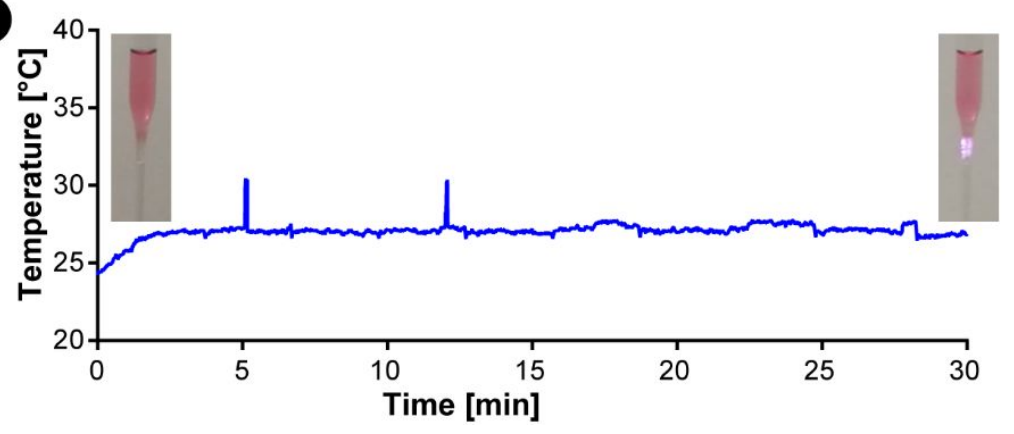

Figure S4: Stability of PVA-NIR785 hydrogels. Blocking setup containing PVA-NIR785 hydrogels $(20 \mu \mathrm{L}$ volume, $20 \mathrm{wt} \%)$ and $0.5 \mathrm{~mL}$ PBS. A) Incubated at $37^{\circ} \mathrm{C}$ for 7 days. B) Incubated at $52^{\circ} \mathrm{C}$ for $22 \mathrm{~h}$. C) PVA- $\mathrm{NH}_{2}$ hydrogel $(20 \mu \mathrm{L}$ volume, $20 \mathrm{wt} \%, 20 \mathrm{kDa})$ irradiated in the blocking setup by NIR light at $1.5 \mathrm{~W}$ for $30 \mathrm{~min}$. Temperature was recorded with an IR-camera. The two spikes in temperature after 5 and $12 \mathrm{~min}$ arise from inspection of the alignment of the sample within the laser beam. Inserts: picture at $\mathrm{t}=0$ and $\mathrm{t}=30 \mathrm{~min}$. 


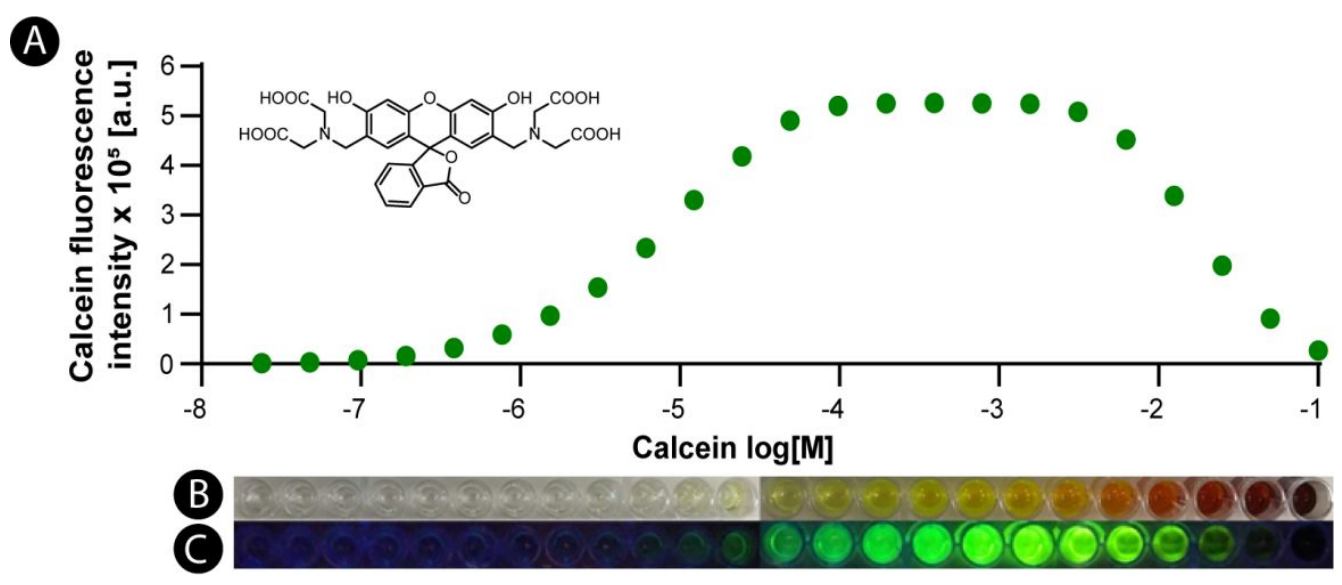

Figure S5: Calcein. A) Self-quenching effect of calcein, shown from a serial dilution of calcein. Fluorescence measurements at $\lambda \mathrm{ex} / \lambda \mathrm{em} 498 / 515 \mathrm{~nm}$. B) Pictures of calcein solutions C) Pictures of calcein fluorescence under a UV lamp, $365 \mathrm{~nm}$.

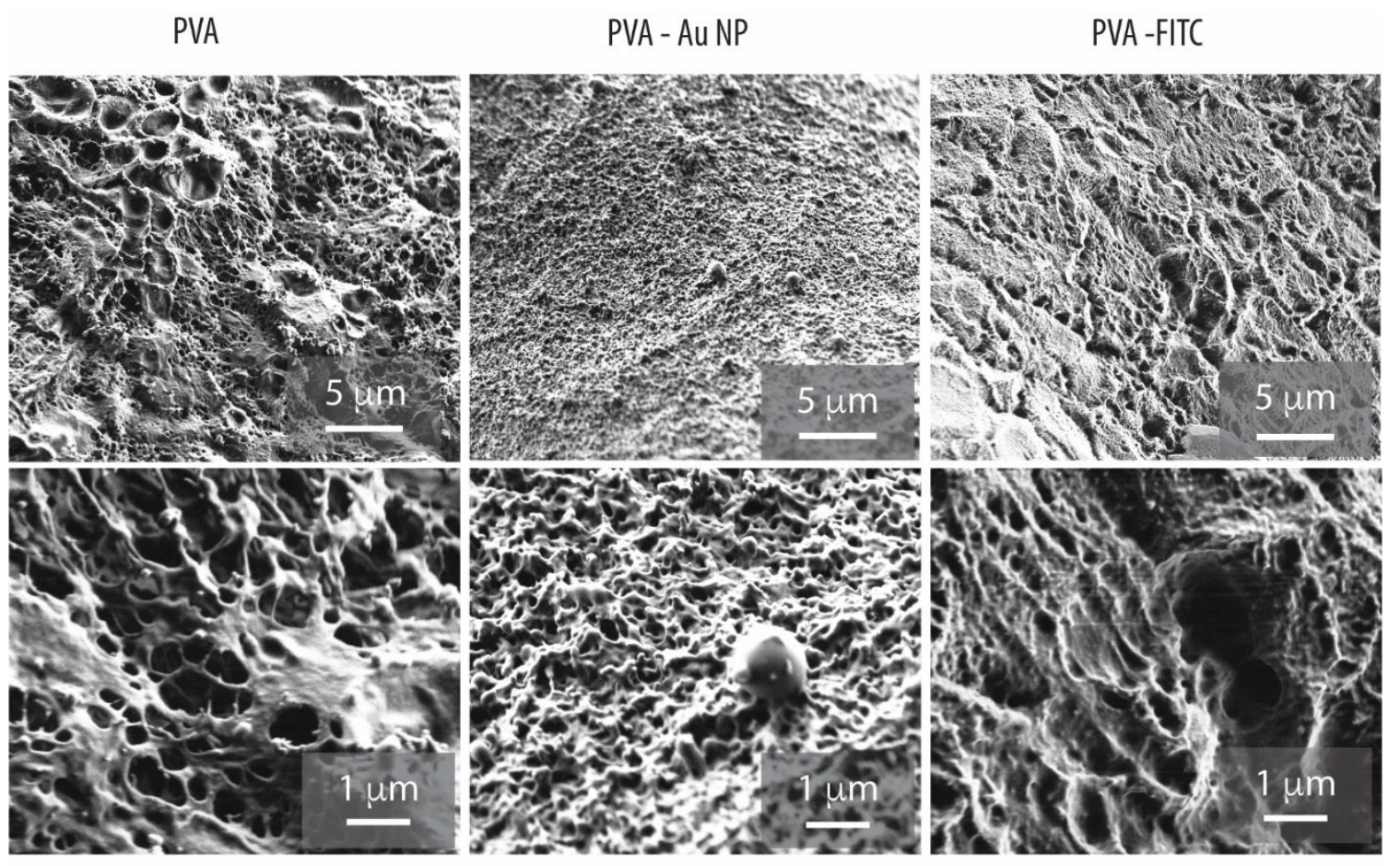

Figure S6. Scanning electron microscopy of PVA hydrogels prepared via critical point drying. 

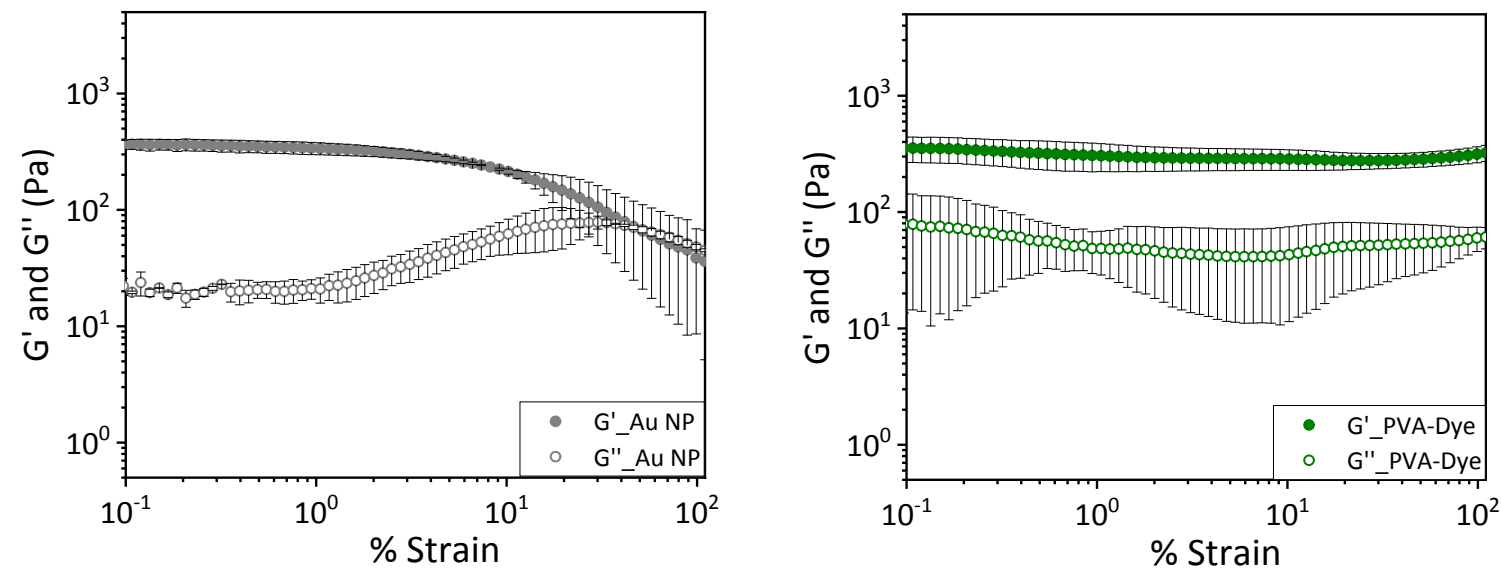

Figure S7: Storage (G', solid circles) and loss modulus (G', open circles) of PVA hydrogels with AuNP (left) and PVA-NIR785 dye conjugate (right) as a function of strain-amplitude sweep at $1 \mathrm{~s}^{-1}$ radial frequency. Data are based on three independent experiments and presented as mean \pm st.dev. 


\section{Super paramagnetic iron oxide nanoparticles and alternating electromagnetic field}

X-ray diffraction was performed on a Rigaku SmartLab II diffractometer. Magnetization measurements were performed on a Physical Properties Measurement System (PPMS) from Quantum Design equipped with a vibrating sample magnetometer (VSM) operating at 300K and in the range of $\pm 20 \mathrm{kOe}$.

All magnetothermal experiments were performed with a MagneTherm ${ }^{\mathrm{TM}}$ system, NanoTherics, UK. The system consists of a high-power radio frequency amplifier connected to a solenoid coil. The radiofrequency amplifier was powered by an external DC power supply, and frequency was adjusted by an external function generator. The system was monitored by an oscilloscope. A double water cooling system with two Julabo bath circulating systems (F250 and ED) was used to cool down the coil and the magneTherm ${ }^{\mathrm{TM}}$ system. The temperature was recorded either with a FLIR C2 Compact Thermal Imaging System and FLIR Tools + software or a thermocouple (EL-USB-TC-LCD data logger) controlled with EasyLog USB software.

Nanoparticle synthesis by reduction of iron chloride salts was performed as in An et al. ${ }^{45}$. In a threenecked flask equipped with a condenser, $\mathrm{FeCl}_{2}$ (430mg, 2.15mmol, 1eq.) and $\mathrm{FeCl}_{3}$ (1189mg, $4.35 \mathrm{mmol}$, 2eq.) were dissolved in degassed $\mathrm{MQ}$ water $(40 \mathrm{~mL})$ at r.t. under $\mathrm{N}_{2}$ atmosphere. Under vigorous stirring $\mathrm{NH}_{4} \mathrm{OH} 28 \%(1.9 \mathrm{~mL}, 12 \mathrm{mmol}$, 5.6eq.) was added rapidly. Stirring rate was lowered and temperature was raised to $80^{\circ} \mathrm{C}$. Oleic acid $\left(150 \mu \mathrm{L}, 0.48 \mathrm{mmol}, 0.22 \mathrm{eq}\right.$.) was added at $50^{\circ} \mathrm{C}$ and the reaction was stirred for $1 \mathrm{~h}$ at $80^{\circ} \mathrm{C}$. Toluene $(30 \mathrm{~mL}$ ) and $\mathrm{NaCl}$ (excess) were added and the two phases were separated. The organic phase was washed with water followed by addition of acetone, which facilitated precipitation and isolation by magnetic decantation. A second wash with toluene and acetone and magnetic decantation yielded $550 \mathrm{mg}$ oleic acid-covered iron oxide particles.

\section{Nanoparticle synthesis by thermal decomposition iron pentacarbonyl.}

SPION were synthesized according to literature ${ }^{46}$. To a three necked $250 \mathrm{~mL}$ round bottomed flask, equipped with a magnetic stirrer and a condenser, was added dioctyl ether $(50 \mathrm{~mL})$ and oleic acid $(6 \mathrm{~mL})$, the solution was heated to $100^{\circ} \mathrm{C}$, degassed and dried of water under a nitrogen flow. Iron pentacarbonyl $(1 \mathrm{~mL})$ was added and temperature was raised to $290^{\circ} \mathrm{C}$ with a ramp of $3^{\circ} \mathrm{C} / \mathrm{min}$. and held at that temperature for $1 \mathrm{~h}$ before returning to r.t. Reaction solution was poured into ethanol $(250 \mathrm{~mL})$ to precipitate SPION which were isolated by magnetic decantation, followed by dispersion in toluene $(6 \mathrm{~mL})$ and reprecipitation in ethanol. SPION were isolated by centrifugation.

\section{Water dispersible SPION, ligand exchange of oleic acid for TMAOH}

SPION (20mg) were washed (magnetic decantation) in ethanol/hexane $(3: 1)$, then mixed with TMAOH $\left(2 \mathrm{~mL}, 25 \mathrm{wt} \%\right.$ in $\left.\mathrm{H}_{2} \mathrm{O}\right)$ and sonicated for $30 \mathrm{~min}$. followed by washes in water yielding $\left(\mathrm{CH}_{3}\right)_{4} \mathrm{~N}^{+}$coated SPION ${ }^{47-48}$. The SPION $(15 \mathrm{mg})$ were subsequently mixed with PVA $(15 \mathrm{mg}, 90 \mathrm{kDa})$ forming a homogeneous aqueous solution ( $8 \mathrm{wt} \%$ SPION and $8 \mathrm{wt} \%$ PVA).

\section{SPION-hydrogel production and treatment in AMF.}

Solutions of PVA and SPION (as presented above with TMAOH) of $8 \mathrm{wt} \%$ SPION and $8 \mathrm{wt} \%$ PVA $(90 \mathrm{kDa})$ was cryogelated as $20 \mu \mathrm{L}$ volume gels using Eppendorfs tubes as molds, five rounds of freeze-thawing. A glass Pasteur pipettes, $150 \mathrm{~mm}$, with a hydrogel and $500 \mu \mathrm{L}$ of water on top was positioned within the AMF (MagneTherm system by NanoTherics) coil and treated up to $30 \mathrm{~min}$ at $476 \mathrm{kHz}$ and $23 \mathrm{mT}$. 

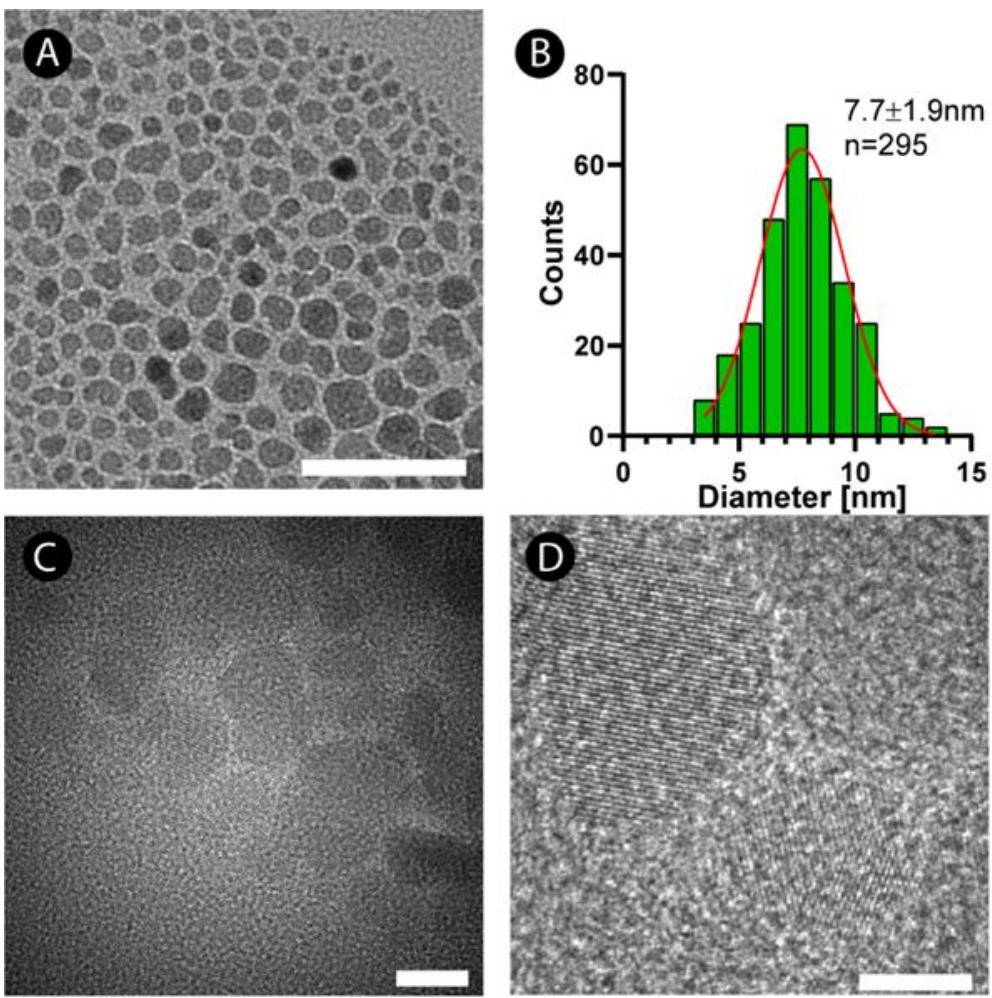

Figure S8: SPION by reduction of iron chloride salts. A) TEM of as synthesized SPION, scale bar: $50 \mathrm{~nm}$. B) Size distribution of SPION based on TEM picture. Diameter: $7.7 \pm 1.9 \mathrm{~nm}(\mathrm{n}=295)$. C) and D) High resolution TEM, scale bar: $10 \mathrm{~nm}$ and $5 \mathrm{~nm}$, respectively.
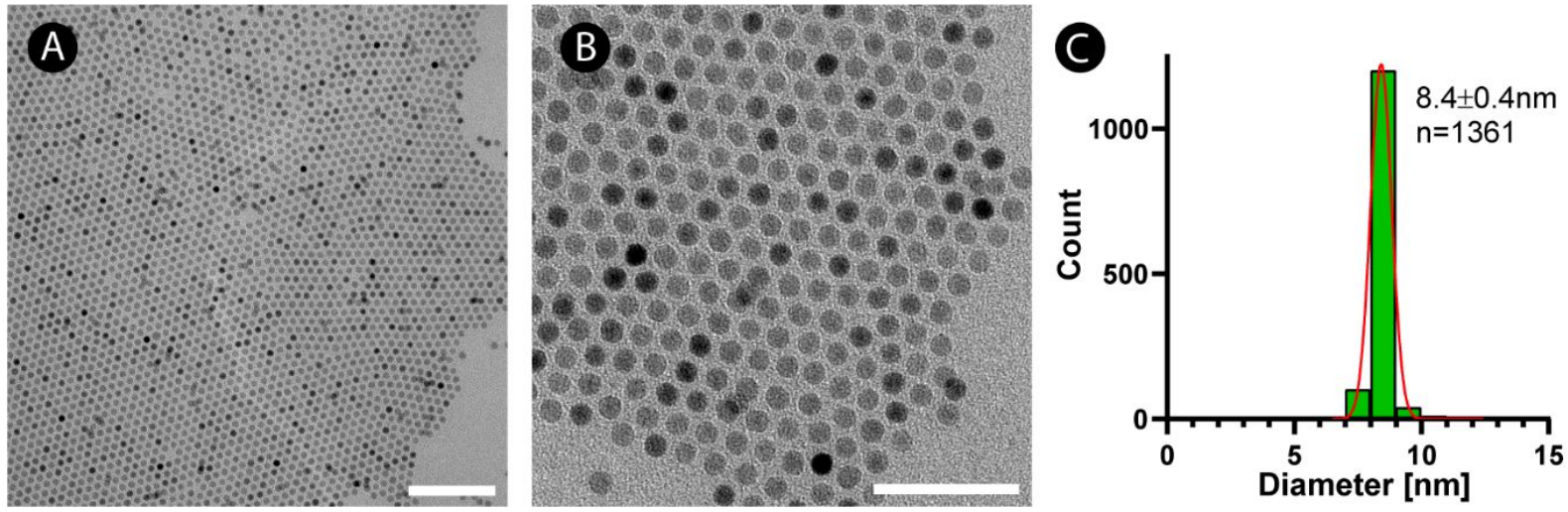

Figure S9: SPION by thermal decomposition of iron pentacarbonyl. A) and B) TEM of as synthesized SPION, scale bar: $100 \mathrm{~nm}$ and $50 \mathrm{~nm}$, respectively. C) Size distribution of SPION based on TEM picture. Diameter: $8.4 \pm 0.4 \mathrm{~nm}(\mathrm{n}=1361)$. 


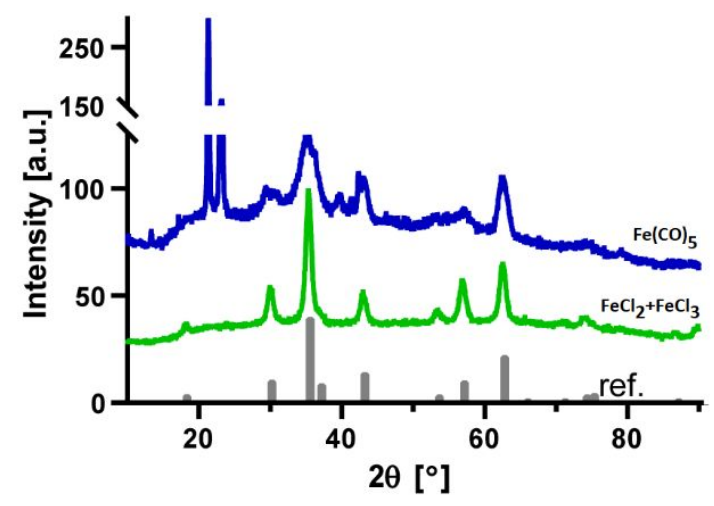

Figure S10: XRD diffractogram of SPION. SPION by reduction of iron chloride salts (green) and SPION by thermal decomposition of iron pentacarbonyl (blue). Reference angles for $\mathrm{Fe}_{3} \mathrm{O}_{4}$ (grey).

A

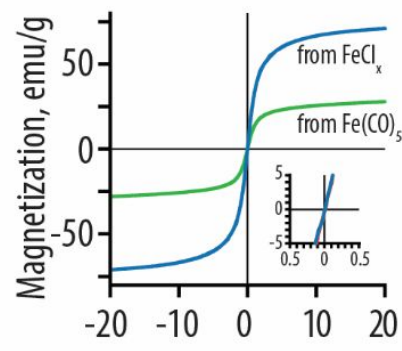

Magnetic Field, $\mathrm{H}$ [kOe]
B

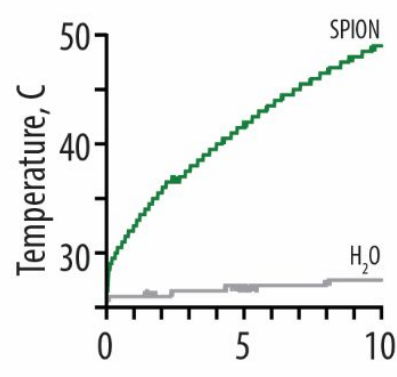

Time, $\min$
C SPION / Alternating EMF

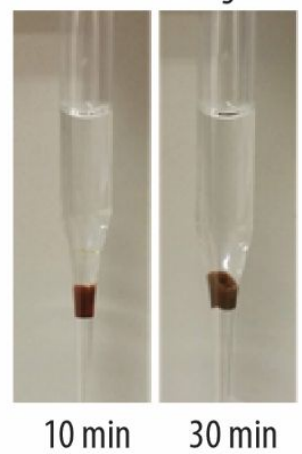

Figure S11. (A) SPION exhibited characteristic magnetisation profile confirming theiradd paramagnetism; (B). Heat evolution profile was recorded in aqueous suspensions of SPION, (C) Visualization of the PVA hydrogels containing SPION following stimulation with AMF $(476 \mathrm{kHz}, 23$ $\mathrm{mT})$. 

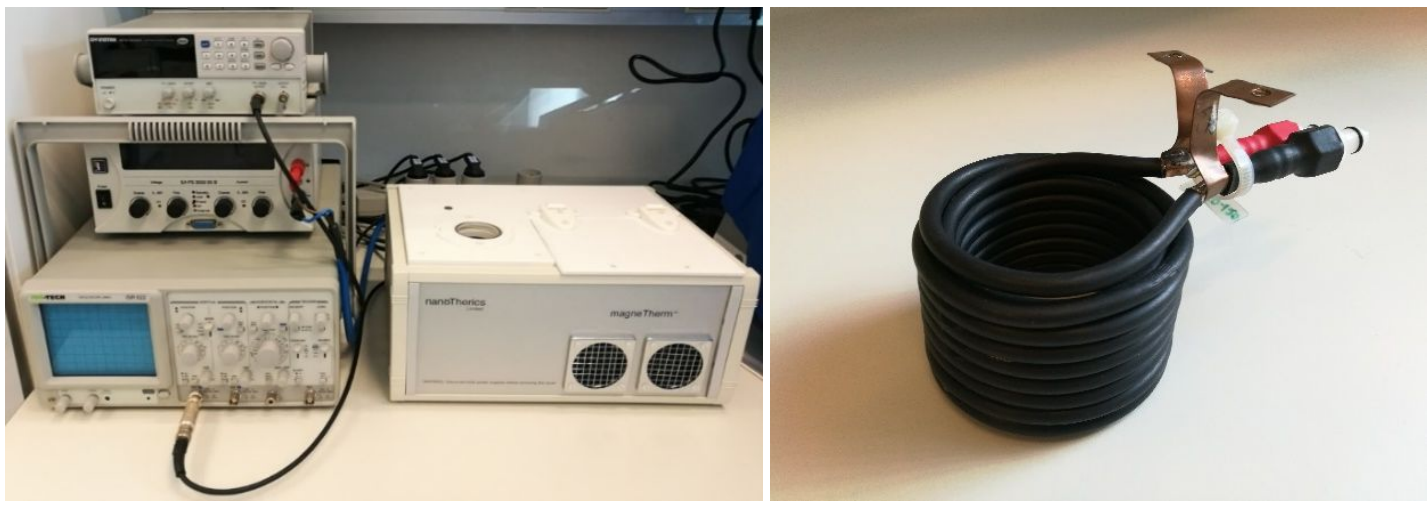

Figure S12: MagneTherm ${ }^{\mathrm{TM}}$ setup. Top left: function generator; middle left: DC power supply; bottom left: oscilloscope; right: magneTherm ${ }^{\mathrm{TM}}$ system containing a solenoid coil (right).

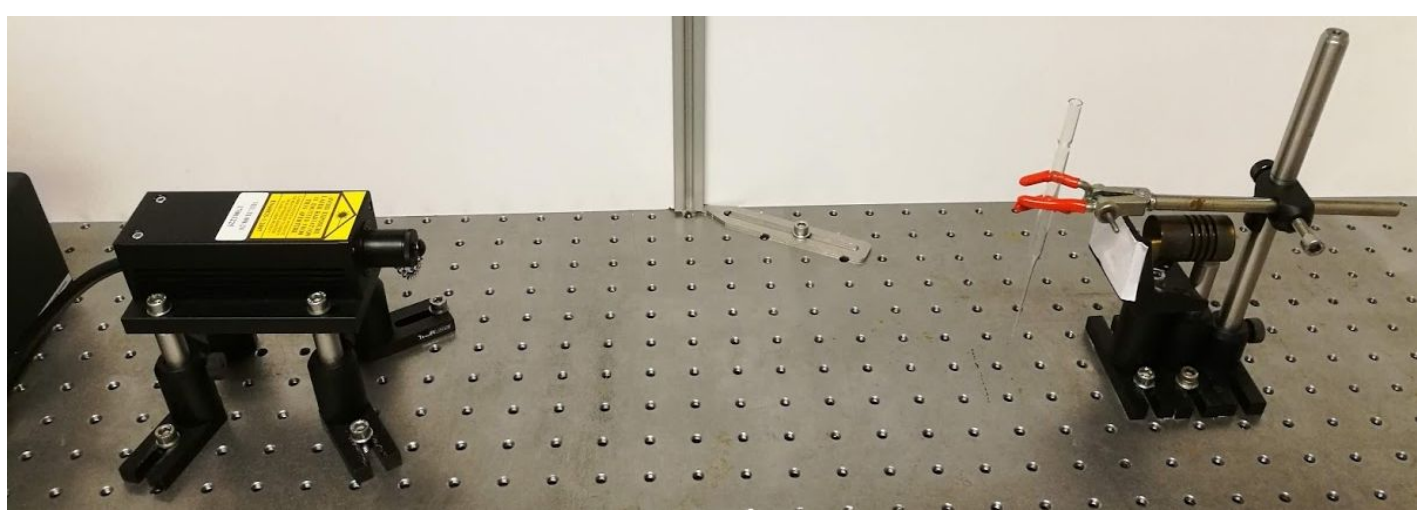

Figure S13: Laser setup, horizontal. NIR light beam aimed for horizontal irradiation.

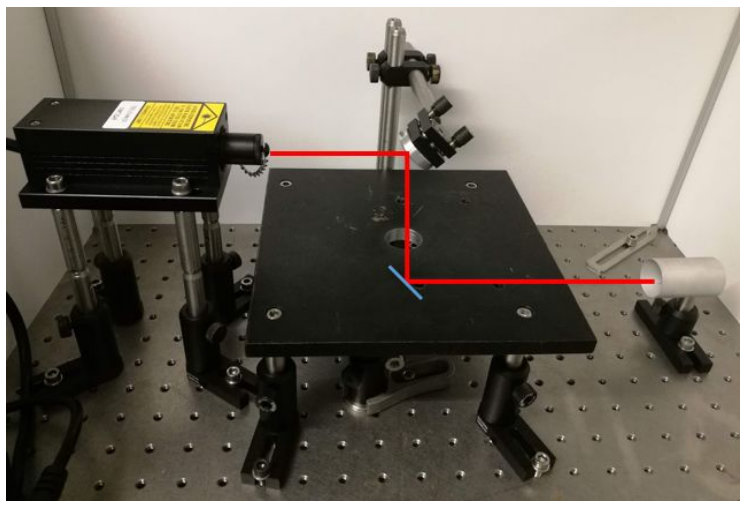

Figure S14: Laser setup, vertical. NIR light beam angled vertically downwards for top-down irradiation. Red line indicate the beam path. 


\section{NMR spectra}
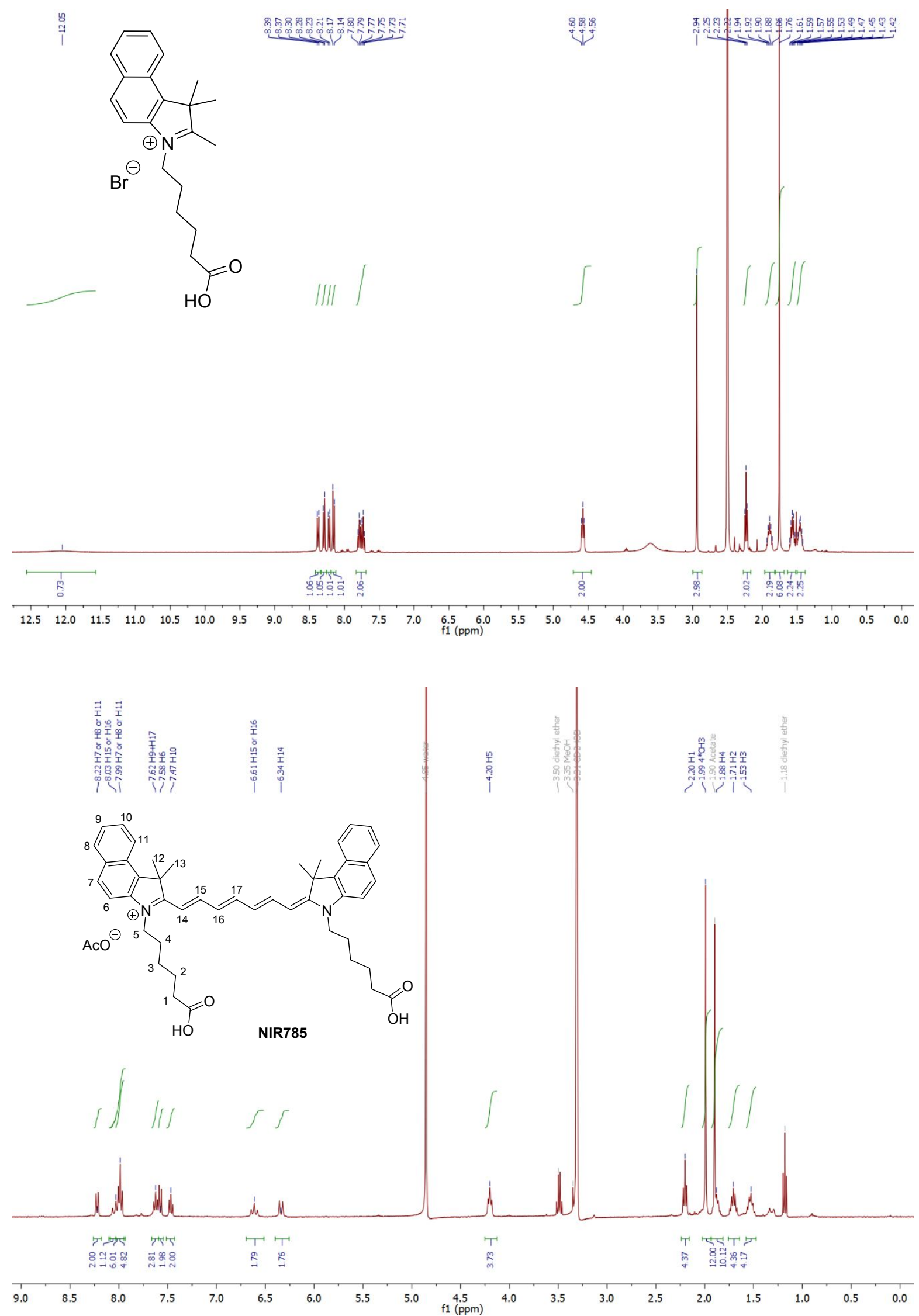


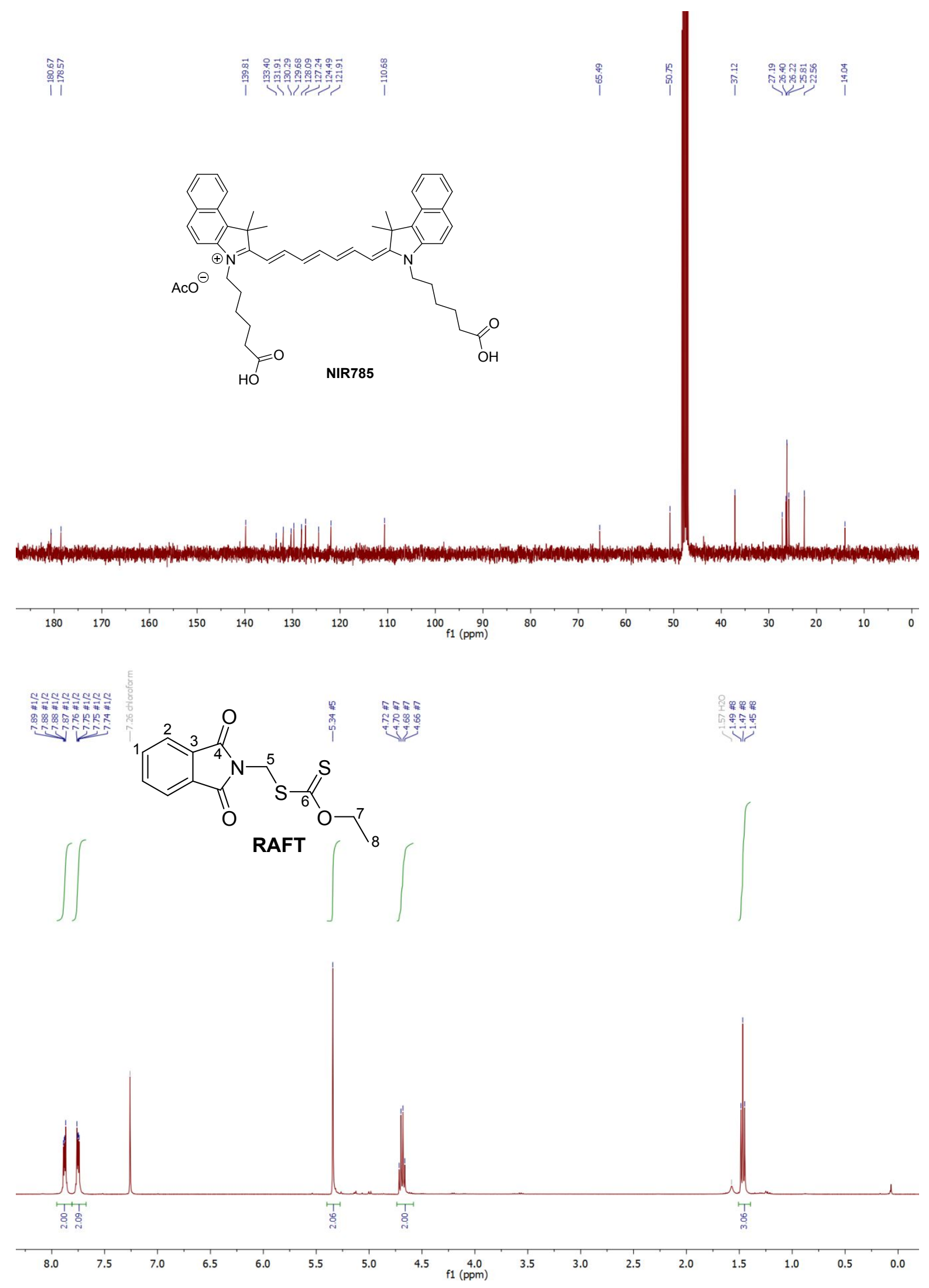




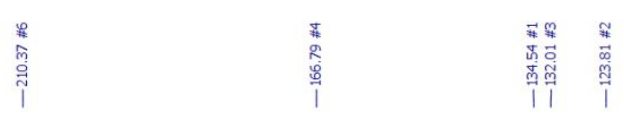

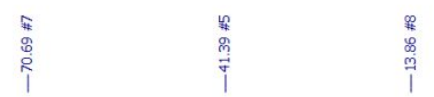<smiles>[R17]COC(=S)SCN1C(=O)c2c[Y]ccc2C1=O</smiles>
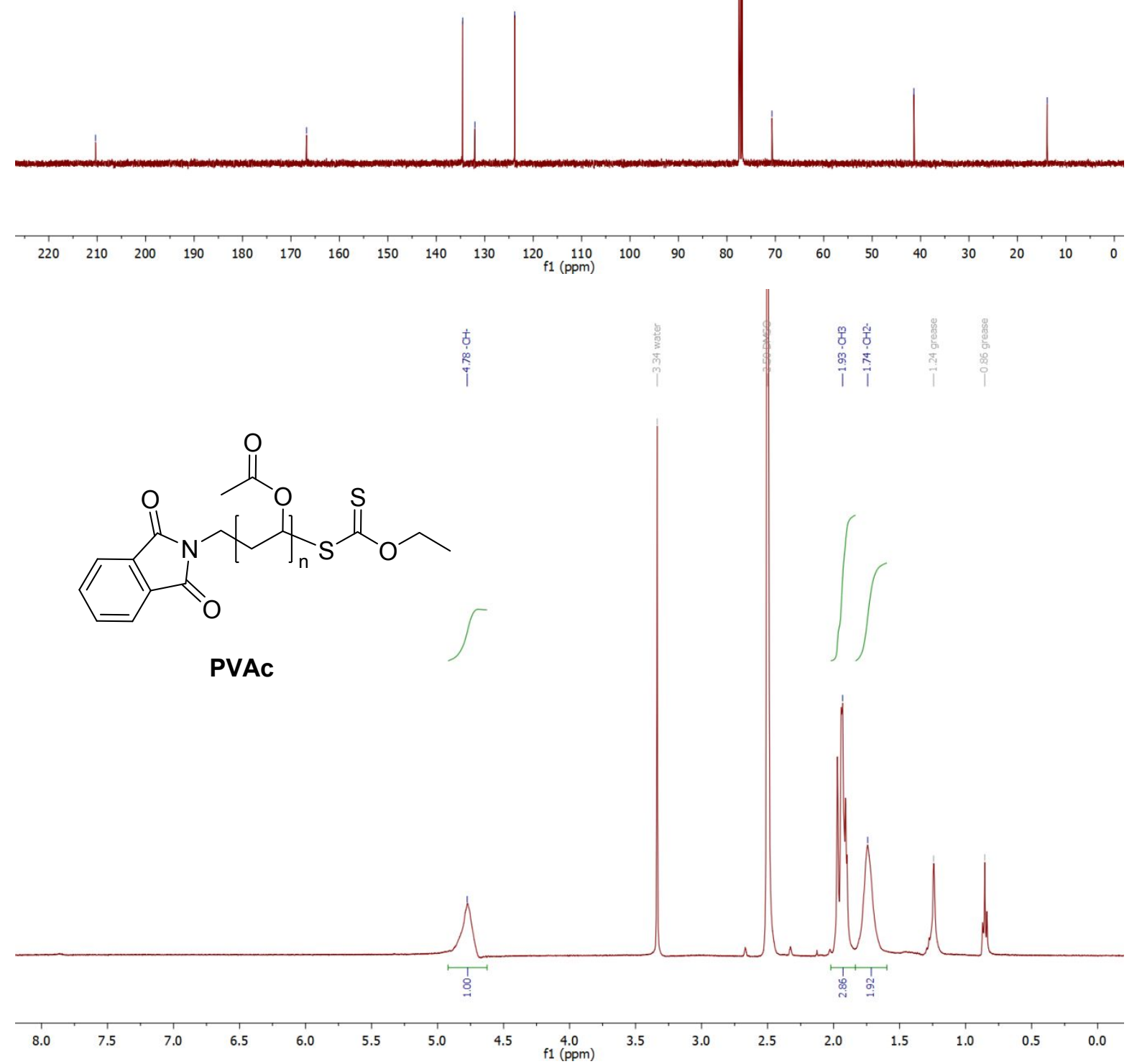


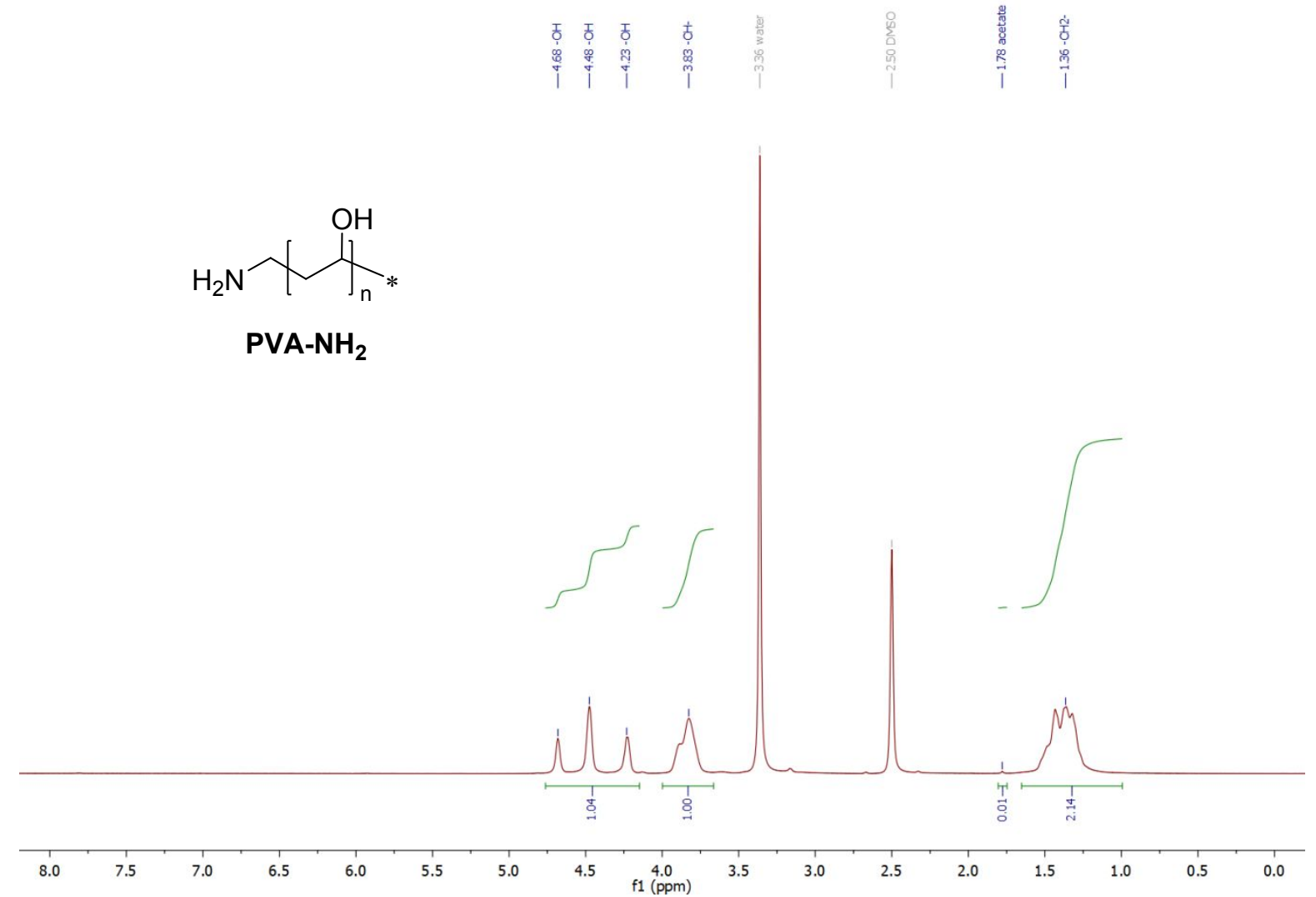

